\title{
Implications of Advanced Method
}

\author{
Meenakshi \\ Assistant Professor , Department of Mathematics, Govind National College, Narangwal, Ludhiana (PB)
}

\begin{abstract}
This paper discusses the implications of Advanced method for finding the optimal solution of transportation problem. Advanced method ${ }^{[2]}$ does not give a solution nearly comparable to MODI method as claimed by Reena G Patel and P.H.Bathwala.In most of the transportation problems difference between the transportation costs given by both the methods are very high.. MODI method proved itself effective invariably in all cases till now. Even in most of the cases VAM gives a better feasible solution than Advanced Method.Two counter examples are given in this paper to prove this point.
\end{abstract}

Keywords: VAM , Transportation problem

\section{Introduction}

As by Hamdy.A.Taha ${ }^{[3]}$,Transportation problem is a special type of LPP where the objective is to minimize the cost of distributing a commodity from fixed number of sources to a fixed number of destinations.Let there are $m$ sources $S_{1}, S_{2}$,

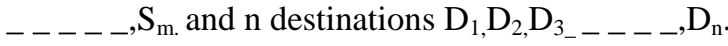

Transportation problem can be represented mathematically as LPP as follows

Minimize : $\mathrm{Z}=$

$$
\sum_{i=1}^{m} \sum_{j=1}^{n} c \mathrm{ij} x \mathrm{ij}
$$

Subject to

$$
\begin{array}{ll}
\sum_{j=1}^{n} \mathrm{xij} \leq \mathrm{ai}, & \mathrm{i}=1,2,3 \ldots . \mathrm{m} \\
\sum_{i=1}^{m} \mathrm{xij} \geq \mathrm{bj}, & \mathrm{j}=1,2,3 \ldots \ldots \mathrm{n} \\
\mathrm{X}_{\mathrm{ij} \geq 0 \text { for all } \mathrm{i}, \mathrm{j}} &
\end{array}
$$

ai = quantity of commodity available at origin $\mathrm{i}$

$\mathrm{bj}=$ requirement of commodity at destination $\mathrm{j}$

cij $=$ cost of transportation of one unit of commodity from ith source to jth destination .

xij = number of units of commodity to be transported from ith source to jth destination

\section{Numerical Examples}

Example 1. Transportation model of problem is given below

\begin{tabular}{l|c|c|c|c|c|c|}
\multicolumn{10}{c|}{ Destinations } \\
\hline Sources & D & E & F & G & supply \\
\hline A & 8 & 10 & 7 & 6 & 50 \\
\hline B & 12 & 9 & 4 & 7 & 40 \\
\hline C & 9 & 11 & 10 & 8 & 30 \\
\hline
\end{tabular}

Solution of the problem by Advanced method is represented in the following table

\begin{tabular}{l}
$\qquad$\begin{tabular}{|c|c|c|c|c|c|}
\hline Sources & D & E & F & G & Supply \\
\hline A & $8_{(10)}$ & 10 & $7_{(40)}$ & 6 & 50 \\
\hline B & 12 & $9_{(17)}$ & 4 & $7_{(23)}$ & 40 \\
\hline C & $9_{(15)}$ & $11_{(15)}$ & 10 & 8 & 30 \\
\hline
\end{tabular} \\
Requirement 25 \\
\hline
\end{tabular}

Total transportation cost

$8 * 10+7 * 40+9 * 17+7 * 23+9 * 15+11 * 15$

$=$ Rs. 974

Solution of transportation problem by VAM is represented in the following table

\begin{tabular}{|c|c|c|c|c|c|}
\hline Sources & $\mathrm{D}$ & $\mathrm{E}$ & $\mathrm{F}$ & $\mathrm{G}$ & Supply \\
\hline A & $8_{(25)}$ & $10_{(2)}$ & 7 & $6_{(23)}$ & 50 \\
\hline B & 12 & 9 & $4_{(40)}$ & 7 & 40 \\
\hline C & 9 & $11_{(30)}$ & 10 & 8 & 30 \\
\hline
\end{tabular}

$\begin{array}{llllll}\text { Requirements } \quad 25 \quad 32 & 40 & 23 & 120\end{array}$

Total transportation cost

$=8 * 25+10 * 2+6 * 23+4 * 40+11 * 30$

=Rs. 848

Solution of problem by MODI method is represented in the following table

\begin{tabular}{|c|c|c|c|c|c|}
\hline Sources & D & E & F & G & Supply \\
\hline A & $8_{(25)}$ & $10_{(2)}$ & 7 & $6_{(23)}$ & 50 \\
\hline B & 12 & 9 & $4_{(40)}$ & 7 & 40 \\
\hline C & 9 & $11_{(30)}$ & 10 & 8 & 30 \\
\hline
\end{tabular}

$\begin{array}{lllllll}\text { Requirements } & 25 & 32 & 40 & 23 & 120 & \\ \text { Total } & & & & & & \text { transportation }\end{array}$ cost $=8 * 25+10 * 2+6 * 23+4 * 40+11 * 30=$ Rs. 848

Example 2: Transportation model of a problem is given below

Destinations

\begin{tabular}{|c|c|c|c|c|c|}
\hline Source & D1 & D2 & D3 & D4 & Supply \\
\hline S1 & 65 & 45 & 35 & 75 & 150 \\
\hline S2 & 60 & 55 & 20 & 80 & 200 \\
\hline S3 & 60 & 50 & 30 & 85 & 130 \\
\hline Req. & 110 & 120 & 150 & 100 & 480 \\
\hline
\end{tabular}

Solution of the problem by Advanced method is represented in the following table.

Destinations

\begin{tabular}{|c|c|c|c|c|c|}
\hline source & D1 & D2 & D3 & D4 & Supply \\
\hline S1 & 65 & 45 & $35_{(50)}$ & $75_{(100)}$ & 150 \\
\hline S2 & 60 & $5_{(100)}$ & $20_{(100)}$ & 80 & 200 \\
\hline S3 & $60_{(110)}$ & $50_{(20)}$ & 30 & 85 & 130 \\
\hline Req. & 110 & 120 & 150 & 100 & 480 \\
\hline
\end{tabular}

Total transportations cost

$=35 * 50+75 * 100+55 * 100+20 * 100+60 * 110+50 * 20$

Volume 6 Issue 1, January 2017 
$=$ Rs. 24,350

Solution of the problem by VAM is reprensented in the following table

\begin{tabular}{|c|c|c|c|c|c|}
\hline Destinations \\
\hline Source & D1 & D2 & D3 & D4 & Supply \\
\hline S1 & 65 & $45_{(120)}$ & 35 & $75_{(30)}$ & 150 \\
\hline S2 & 60 & 55 & $20_{(150)}$ & $80_{(50)}$ & 200 \\
\hline S3 & $60_{(110)}$ & 50 & 30 & $85_{(20)}$ & 130 \\
\hline Req. & 110 & 120 & 150 & 100 & 480 \\
\hline
\end{tabular}

Total transportation cost $=$

$45 * 120+75 * 30+20 * 150+80 * 50+60 * 110+85 * 20$

$=$ Rs22,950

Solution of the problem by MODI method is represented in the following table

\begin{tabular}{|c|c|c|c|c|c|}
\hline Source & D1 & D2 & D3 & D4 & Supply \\
\hline S1 & 65 & $45_{(100)}$ & 35 & $75_{(50)}$ & 150 \\
\hline S2 & 60 & 55 & $20_{(150)}$ & $80_{(50)}$ & 200 \\
\hline S3 & $60_{(110)}$ & $50_{(20)}$ & 30 & 85 & 130 \\
\hline Req. & 110 & 120 & 150 & 100 & 480 \\
\hline
\end{tabular}

Total transportation cost $=$

$45 * 100+75 * 50+20 * 150+80 * 50+60 * 110+50 * 20=\mathrm{Rs} 22,850$

\section{Result Analysis}

Above examples contradicts the claim of Reena.G.Patel and P.H.Bhathwala. The comparison table of the solutions given by Advanced,VAM and MODI methods is given below.

\begin{tabular}{|c|c|c|}
\hline Methods & \multicolumn{2}{|c|}{$\begin{array}{c}\text { Total transportation cost(in Rs) } \\
\text { Example1 }\end{array}$} \\
\hline Example2 \\
\hline Advanced & 974 & 24,350 \\
\hline MOM & 848 & 22,950 \\
\hline
\end{tabular}

\section{Conclusion}

Above noted study proves that Advanced method is not comparable to MODI method for finding optimal solution of a transportation problem.

\section{References}

[1] Dantzig G.B ,Linear programming and extentions. Princeton university press,Princeton,NJ,1963.

[2] Patel.Reena.G,Bhathwala.P.H , Advanced Method for the Optimum solution of a transportation problem, International Journal of Science and Research,23197064,2015

[3] Hamdy A.T. , operations Research: An introduction, $8^{\text {th }}$ edition, Pearson Prentice Hall,New Delhi,2007.

[4] Hadley.G,Linear Programming,Narosa Publishing House, $6^{\text {th }}$ edition, 2006. 